\title{
Asymptotically Flat Radiating Solutions in Third Order Lovelock Gravity
}

\author{
M. H. Dehghani ${ }^{1,2 *}$, N. Farhangkhah ${ }^{1}$ \\ ${ }^{1}$ Physics Department and Biruni Observatory, \\ College of Sciences, Shiraz University, Shiraz 71454, Iran \\ ${ }^{2}$ Research Institute for Astrophysics and Astronomy of Maragha (RIAAM), Maragha, Iran
}

\begin{abstract}
In this paper, we present an exact spherically symmetric solution of third order Lovelock gravity in $n$ dimensions which describes the gravitational collapse of a null dust fluid. This solution is asymptotically (anti-)de Sitter or flat depending on the choice of the cosmological constant. Using the asymptotically flat solution for $n \geq 7$ with a power-law form of the mass as a function of the null coordinate, we present a model for a gravitational collapse in which a null dust fluid radially injects into an initially flat and empty region. It is found that a naked singularity is inevitably formed whose strength is different for the $n=7$ and $n \geq 8$ cases. In the $n=7$ case, the limiting focusing condition for the strength of curvature singularity is satisfied. But for $n \geq 8$, the strength of curvature singularity depends on the rate of increase of mass of the spacetime. These considerations show that the third order Lovelock term weakens the strength of the curvature singularity.
\end{abstract}

* email address: mhd@shirazu.ac.ir 


\section{INTRODUCTION}

The problem of gravitational collapse in general relativity is one of the unsolved problems in gravitation physics. One would like to know whether, and under what initial conditions, gravitational collapse results in black hole formation. In particular, one would like to know if there are physical collapse solutions that lead to naked singularities. In 1939, Oppenheimer and Snyder [1] studied, as an idealized model of gravitational collapse, the solution which corresponds to a homogeneous spherically symmetric dust cloud. By analyzing the behavior of the outgoing light rays they were led to black hole idea. In general relativity, singularity theorems were proved stating that spacetime singularities inevitably appear under general situations and physical energy conditions [2]. However, the nature of the singularities remains an outstanding unresolved question. In this context, a cosmic censorship hypothesis (CCH) was proposed by Penrose, which states that curvature singularities in asymptotically flat spacetimes are always shrouded by event horizons; in other words, there are no naked singularities formed in physical gravitational collapse [3]. The weak $\mathrm{CCH}$ allows for the occurrence of locally naked singularities but not globally naked ones, whereas the strong $\mathrm{CCH}$ does not allow either. However, there are a number of important results which assume the truth of this hypothesis, such as the area theorem of black holes, and soon after it some counterexamples to this hypothesis were found. One of those is the generic occurrence of naked singularities in the null dust collapse shown by Vaidya. In 1959, Vaidya found a solution that represents an imploding (exploding) null dust fluid with spherical symmetry [4]. Since then this solution has been studied in gravitational collapses by many authors. In particular, it has been showed that this solution can give rise to the formation of the naked singularity, and after that some other counterexamples to the cosmic censorship hypothesis were provided [5].

There exist many possible generalizations of the Vaidya-type metric. The (anti-)de Sitter [(A)dS] Vaidya-type solutions in Einstein gravity have been worked widely until now in [6] and the references therein. In these papers, the asymptotically (A)dS spherically symmetric Vaidya-type solutions of Einstein gravity with cosmological constant have been found, and the existence of naked singularity has been investigated. These papers show that whether the spacetime is asymptotically flat or not has no any effect on the occurrence of a locally naked singularity. Also the effects of the dimensionality of spacetime on the weak CCH has 
been investigated [7].

In recent years a renewed interest has grown in higher-order gravity, which involves higher derivative curvature terms. Among them, the second order Lovelock gravity or the so-called Einstein-Gauss-Bonnet gravity and the third order Lovelock gravity are of particular interest because of their special features. Exact solutions of the former can be found in [8, 9] and the latter in [10, 11]. Also, the possibility of a dark energy universe emerging from an action with higher-order curvature terms has been investigated [12]. Here we want to investigate the effects of higher-order Lovelock terms on $\mathrm{CCH}$ for those solutions which have a general relativistic limit. The effects of second order Lovelock terms on the Vaidya-type solutions have been investigated in [13, 14]. These papers show that the appearance of a second order Lovelock term in the field equations has no effect on the occurrence of locally naked singularity, while it has some effects on the strength of the curvature. In this paper, our aim is to consider the effect of the third order Lovelock term on the gravitational collapse of the null fluid, the occurrence of a naked singularity, and the strength of the curvature. The Vaidya-type solution of dimensionally continued Lovelock gravity has been found in [15]. This solution is asymptotically AdS and contains only one fundamental constant. Indeed, this solution cannot show the effect of higher-order Lovelock terms explicitly on the weak $\mathrm{CCH}$, which is proposed for asymptotically flat spacetimes of Einstein gravity. Here we find asymptotically flat and (A)dS Vaidya-type solutions of third order Lovelock gravity with four fundamental constants (the cosmological constant and the three Lovelock coefficients).

The outline of our paper is as follows. In Sec. II, we introduce the Vaidya-type solutions of third order Lovelock gravity and obtain the $n$-dimensional solution of a spacetime outside a radiating star with the energy-momentum tensor of a null fluid. In Sec. III, we show that the final fate of this spacetime is a naked singularity. Section IV is devoted to the investigation of the strength of the curvature singularity for different rates of increase of the mass of the spacetime in an arbitrary dimension. We finish our paper with some concluding remarks.

\section{VAIDYA-TYPE SOLUTION IN THIRD ORDER LOVELOCK GRAVITY}

A natural generalization of general relativity in higher-dimensional spacetimes with the assumption of Einstein, that the left hand side of the field equations is the most general 
symmetric conserved tensor containing no more than second derivatives of the metric, is the Lovelock theory. The gravitational field equations of third order Lovelock gravity may be written as

$$
\Lambda^{\prime} g_{\mu \nu}+G_{\mu \nu}^{(1)}+\alpha_{2}^{\prime} G_{\mu \nu}^{(2)}+\alpha_{3}^{\prime} G_{\mu \nu}^{(3)}=\kappa_{n}^{2} T_{\mu \nu}
$$

where $\Lambda^{\prime}$ is the cosmological constant, $\alpha_{i}^{\prime}$ 's are Lovelock coefficients which are assumed to be positive throughout the paper, $T_{\mu \nu}$ is the energy-momentum tensor of the matter, $G_{\mu \nu}^{(1)}$ is the Einstein tensor, and $G_{\mu \nu}^{(2)}$ and $G_{\mu \nu}^{(3)}$ are the second and third order Lovelock tensors, respectively, given as

$$
\begin{aligned}
& G_{\mu \nu}^{(2)}=2\left(R_{\mu \sigma \kappa \tau} R_{\nu}{ }^{\sigma \kappa \tau}-2 R_{\mu \rho \nu \sigma} R^{\rho \sigma}-2 R_{\mu \sigma} R^{\sigma}{ }_{\nu}+R R_{\mu \nu}\right)-\frac{1}{2} \mathcal{L}_{2} g_{\mu \nu}, \\
& G_{\mu \nu}^{(3)}=-3\left(4 R^{\tau \rho \sigma \kappa} R_{\sigma \kappa \lambda \rho} R_{\nu \tau \mu}^{\lambda}-8 R_{\lambda \sigma}^{\tau \rho} R_{\tau \mu}^{\sigma \kappa} R_{\nu \rho \kappa}^{\lambda}+2 R_{\nu}^{\tau \sigma \kappa} R_{\sigma \kappa \lambda \rho} R^{\lambda \rho}{ }_{\tau \mu}\right. \\
& -R^{\tau \rho \sigma \kappa} R_{\sigma \kappa \tau \rho} R_{\nu \mu}+8 R_{\nu \sigma \rho}^{\tau} R_{\tau \mu}^{\sigma \kappa} R_{\kappa}^{\rho}+8 R_{\nu \tau \kappa}^{\sigma} R_{\sigma \mu}^{\tau \rho} R_{\rho}^{\kappa} \\
& +4 R_{\nu}{ }^{\tau \sigma \kappa} R_{\sigma \kappa \mu \rho} R_{\tau}^{\rho}-4 R_{\nu}{ }^{\tau \sigma \kappa} R_{\sigma \kappa \tau \rho} R_{\mu}^{\rho}+4 R^{\tau \rho \sigma \kappa} R_{\sigma \kappa \tau \mu} R_{\nu \rho}+2 R R_{\nu}{ }^{\kappa \tau \rho} R_{\tau \rho \kappa \mu} \\
& +8 R_{\nu \mu \rho}^{\tau} R_{\sigma}^{\rho} R_{\tau}^{\sigma}-8 R_{\nu \tau \rho}^{\sigma} R_{\sigma}^{\tau} R_{\mu}^{\rho}-8 R_{\sigma \mu}^{\tau \rho} R_{\tau}^{\sigma} R_{\nu \rho}-4 R R_{\nu \mu \rho}^{\tau} R_{\tau}^{\rho} \\
& \left.+4 R^{\tau \rho} R_{\rho \tau} R_{\nu \mu}-8 R_{\nu}^{\tau} R_{\tau \rho} R_{\mu}^{\rho}+4 R R_{\nu \rho} R_{\mu}^{\rho}-R^{2} R_{\nu \mu}\right)-\frac{1}{2} \mathcal{L}_{3} g_{\mu \nu} .
\end{aligned}
$$

In Eqs. (21) and (3) $\mathcal{L}_{2}=R_{\mu \nu \gamma \delta} R^{\mu \nu \gamma \delta}-4 R_{\mu \nu} R^{\mu \nu}+R^{2}$ is the Gauss-Bonnet Lagrangian and

$$
\begin{aligned}
\mathcal{L}_{3}= & 2 R^{\mu \nu \sigma \kappa} R_{\sigma \kappa \rho \tau} R_{\mu \nu}^{\rho \tau}+8 R_{\sigma \rho}^{\mu \nu} R_{\nu \tau}^{\sigma \kappa} R_{\mu \kappa}^{\rho \tau}+24 R^{\mu \nu \sigma \kappa} R_{\sigma \kappa \nu \rho} R_{\mu}^{\rho} \\
& +3 R R^{\mu \nu \sigma \kappa} R_{\sigma \kappa \mu \nu}+24 R^{\mu \nu \sigma \kappa} R_{\sigma \mu} R_{\kappa \nu}+16 R^{\mu \nu} R_{\nu \sigma} R_{\mu}^{\sigma}-12 R R^{\mu \nu} R_{\mu \nu}+R^{3}
\end{aligned}
$$

is the third order Lovelock Lagrangian. In order to have the contribution of all of the above terms in the field equation, the dimension of the spacetime should be equal or larger than 7.

Here we are looking for Vaidya-type solutions of third order Lovelock gravity. Thus, the only nonvanishing component of the energy-momentum tensor is $T_{v}{ }^{r}$. The metric of the $n$-dimensional spherically symmetric Vaidya-type solution may be written as

$$
d s^{2}=-f(r, v) d v^{2}+2 \epsilon d r d v+r^{2} d \Omega_{n-2}^{2}
$$

where $v \in(-\infty,+\infty)$ is a null coordinate which represents advanced Eddington time and is ingoing (outgoing) for $\epsilon=+1(-1), 0 \leq r<\infty$ is the radial coordinate, and $d \Omega_{n-2}^{2}$ is the 
line element of the $(n-2)$-dimensional unit sphere:

$$
d \Omega_{n-2}^{2}=d \theta_{1}^{2}+\sum_{i=2}^{n-2} \prod_{j=1}^{i-1} \sin ^{2} \theta_{j} d \theta_{i}^{2} .
$$

We want to obtain the Vaidya-type solutions of third order Lovelock gravity. In this case, the energy-momentum tensor for the directional flow of radiation in empty space is

$$
T_{a}{ }^{b}=\sigma(r, v) k_{a} k^{b}
$$

where $k_{a}=-\partial_{a} v$ and $\sigma(r, v)$ is the density of flowing radiation. The only nonvanishing component of $T_{a}{ }^{b}$ is $T_{v}{ }^{r}$, and therefore the field equations (11) reduce to the following differential equations for $f(r, v)$ :

$$
\begin{aligned}
& (n-1) \Lambda r^{6}+\left\{\alpha_{3} r[f(r, v)-1]^{2}-2 \alpha_{2} r^{3}[f(r, v)-1]+r^{5}\right\} f^{\prime}+ \\
& \frac{n-7}{3} \alpha_{3}[f(r, v)-1]^{3}-(n-5) \alpha_{2} r^{2}[f(r, v)-1]^{2}+(n-3) r^{4}[f(r, v)-1]=0, \\
& -\frac{(n-2)}{2 r^{5}}\left\{\alpha_{3}[f(r, v)-1]^{2}-2 \alpha_{2} r^{2}[f(r, v)-1]+r^{4}\right\} \dot{f}=\kappa_{n}^{2} T_{v}{ }^{r},
\end{aligned}
$$

where the prime and the dot denote the derivatives with respect to the coordinates $r$ and $v$, respectively and we define $\Lambda^{\prime}=2 \Lambda /(n-1)(n-2), \alpha_{2}^{\prime}=\alpha_{2} /(n-3)(n-4)$, and $\alpha_{3}^{\prime}=$ $\alpha_{3} / 3(n-3) \ldots(n-6)$ for simplicity. Equation (7) has one real and two complex solutions. The only real solution of Eq. (17) which reduces to the solution of Einstein gravity in the limit of $\alpha_{2}=\alpha_{3}=0$ can be written as

$$
\begin{aligned}
& f(r, v)=1+\frac{\alpha_{2} r^{2}}{\alpha_{3}}\left\{1-C^{1 / 3}(r, v)+\gamma^{1 / 3} C^{-1 / 3}(r, v)\right\} \\
& C(r, v)=\left(\sqrt{\gamma+k^{2}(r, v)}+k(r, v)\right) \\
& k(r, v)=-1+\frac{3 \alpha_{3}}{2 \alpha_{2}^{2}}+\frac{3 \Lambda \alpha_{3}^{2}}{2 \alpha_{2}^{3}}+\frac{3 \alpha_{3}^{2}}{2 \alpha_{2}^{3}} \frac{M(v)}{r^{n-1}}, \quad \gamma=\left(\frac{\alpha_{3}-\alpha_{2}^{2}}{\alpha_{2}^{2}}\right)^{3},
\end{aligned}
$$

where $M(v)$ is the measure of the mass of the spacetime (see Appendix A). One should note that $\gamma+k^{2}(r, v)$ is larger than $\gamma+k^{2}(\infty, v)$, and the latter should be positive or at least zero due to the reality of the metric function $f(v, r)$ everywhere. This concludes that $\alpha_{3}>3 \alpha_{2}^{2} / 4$. The metric given in Eqs. (5) and (9) is asymptotically flat for $\Lambda=0$ and AdS and $\mathrm{dS}$ for negative and positive values of $\Lambda$, respectively. In this paper, we are interested in asymptotically flat Vaidya-type solutions with a general relativistic limit in order to consider 
$\mathrm{CCH}$ in higher-order Lovelock gravity. This solution reduces to an $n$-dimensional Vaidyatype solution as $\alpha_{2}$ and $\alpha_{3}$ vanish [4]. The case that $M=$ const. reduces to the static solution of third order Lovelock gravity, which is considered in [10].

The only nonvanishing component of stress energy tensor can be found from Eq. (8) as

$$
T_{v}{ }^{r}=\frac{(n-2)}{2 \kappa_{n}^{2} r^{n-2}} \dot{M} .
$$

It is worthwhile to note that the dependence of the energy-momentum tensor given in Eq. (10) on $v$ and $r$ is the same as that of Vaidya-type solutions of Einstein [7], Gauss-Bonnet [13] or dimensionally continued Lovelock [15] gravities.

\section{THE EXISTENCE AND NATURE OF A NAKED SINGULARITY}

In this and the next sections, we study the gravitational collapse of a null dust fluid in third order Lovelock gravity and compare it with that in Einstein relativity [7]. In order to investigate $\mathrm{CCH}$ in third order Lovelock gravity, we consider asymptotically flat solutions $(\Lambda=0)$. The physical situation here is that of a radial influx of null fluid in an initially

empty region of the higher-dimensional Minkowskian spacetime. The first shell arrives at $r=0$ at time $v=0$ and the final shell at $v=v_{f}$. A central singularity of growing mass is developed at $r=0$. For $v<0$, we have $m(v)=0$, i.e., higher-dimensional Minkowskian spacetime, and for $v>v_{f}, M(v)=M_{f}$ is a positive constant, and we have the static solution of third order Lovelock gravity considered in [10]. We choose a power-law growth of mass as

$$
M(v)= \begin{cases}0 & v<0 \\ M_{0} v^{p} & 0 \leq v \leq v_{f} \\ M=M_{0} v_{f}^{p} & v>v_{f}\end{cases}
$$

In order to consider the existence of a physical singularity, we compute the Kretschmann scalar. It is a matter of calculations to show that the Kretschmann scalar diverges for $r \rightarrow 0$ as

$$
K=O\left(\frac{v^{2 p / 3}}{r^{2(n-1) / 3}}\right),
$$

which shows that there is a singularity at $r=0$ for $v \geq 0$.

The nature of the singularity (to be naked or hidden) can be characterized by the existence of radial null geodesics coming out of the singularity. It can be shown that if a futuredirected radial null geodesic does not emanate from the singularity, a future-directed causal 
(excluding radial null) geodesic does not, too [15]. So we consider here only the futuredirected outgoing radial null geodesics which satisfies

$$
\frac{d r}{d v}=\frac{f}{2}
$$

The region with $f<0$ is the trapped region, and a hypersurface $f=0$ represents the trapping horizon [16]. The radius of the apparent horizon as a function of $v$ is given by the following equation:

$$
M(v)=M_{0} v^{p}=\frac{\alpha_{3}}{3} r^{n-7}+\alpha_{2} r^{n-5}+r^{n-3} .
$$

Along the trapping horizon we have:

$$
d s^{2}=\frac{6 p M_{0} v^{p-1}}{(n-7) \alpha_{3} r^{n-8}+3(n-5) \alpha_{2} r^{n-6}+3(n-3) r^{n-4}} d v^{2},
$$

and hence it is spacelike for $v>0$ and $r>0$ for positive Lovelock coefficients. It is a future outer trapping horizon, which is a local definition of black hole (see Appendix B). It is seen from (12) that only the point $r=v=0$ may be a naked singularity for $n \geq 8$, while the central singularity $(r=0, v>0)$ is naked for $0 \leq v \leq v_{a h}$ in the case of $n=7$, where $v_{a h}$ is given as

$$
3 M_{0} v_{a h}^{p}=\alpha_{3} .
$$

In order to show the existence of a null geodesic emanating from the singularity, we adopt the fixed-point method [17]. We introduce a new coordinate $\vartheta=r /\left(v-v_{0}\right)$, where $v_{0}$ is a constant which is in the range $0 \leq v_{0} \leq v_{a h}$ when $n=7$, while it is zero for $n \geq 8$. Then the null geodesic equation (11) becomes

$$
\frac{d r}{d v}=\frac{d \vartheta}{d v}\left(v-v_{0}\right)+\vartheta=\frac{1}{2}\left\{1+\frac{\alpha_{2} r^{2}}{\alpha_{3}}\left[1-C(v, r)^{1 / 3}+\gamma^{1 / 3} C(v, r)^{-1 / 3}\right]\right\},
$$

which may be written as

$$
\frac{d \vartheta}{d v}+\frac{1}{\left(v-v_{0}\right)}(\vartheta-\eta)=\eta \Psi
$$

where we have introduced the parameter $\eta$ in the range $0<\eta<\infty$ and $\Psi$ is

$$
\Psi=-\frac{1}{\left(v-v_{0}\right)}+\frac{1}{2 \eta\left(v-v_{0}\right)}\left\{1+\frac{\alpha_{2}\left(\vartheta\left(v-v_{0}\right)\right)^{2}}{\alpha_{3}}\left[1-C(v, r)^{1 / 3}+\gamma^{1 / 3} C(v, r)^{-1 / 3}\right]\right\} .
$$

If $\eta$ is chosen to be

$$
\eta_{0}=\frac{1}{2}\left\{1-\left(\frac{3 M_{0} v_{0}^{p}}{\alpha_{3}}\right)^{1 / 3}\right\}
$$


for $n \geq 7, \Psi$ is at least $C^{1}$ for $v \geq v_{0}$ and $\vartheta>0$. In this case, Eq. (16) has a nonnegative unique solution which satisfies $\vartheta\left(v_{0}\right)=\eta_{0}$ and is continuous at $v=v_{0}$ [17]. This solution represents an outgoing light ray which emanates from the singular point at the center and which either intersects the apparent horizon or goes to future null infinity [17]. Thus, the solution may be interpreted as a naked singularity.

\section{STRENGTH OF THE SINGULARITY}

The strength of a singularity, which is the measure of its destructive capacity, is the most important feature. Following the paper [18] we consider the null geodesics affinely parametrized by $\lambda$ and terminating at a shell focusing singularity $r=v=\lambda=0$. Then the solution satisfies a strong curvature condition (SCC) [19] if

$$
\lim _{\lambda \rightarrow 0} \lambda^{2} \Phi>0
$$

and limiting focusing condition (LFC) [20] if

$$
\lim _{\lambda \rightarrow 0} \lambda \Phi>0
$$

where

$$
\Phi \equiv R_{\mu \nu} k^{\mu} k^{\nu}
$$

Using the fact that $d r / d \lambda=(d v / d \lambda) f / 2$, one can show that

$$
\Phi=-\frac{2(n-2) \dot{f}}{r f^{2}}\left(\frac{d r}{d \lambda}\right)^{2},
$$

and the radial null geodesic satisfies the differential equation

$$
\frac{d^{2} r}{d \lambda^{2}} \simeq \frac{2 \dot{f}}{f^{2}}\left(\frac{d r}{d \lambda}\right)^{2}
$$

\section{A. $p=n-3$ case:}

In this subsection, in order to compare our results with the results of the curvature strength of $n$-dimensional solutions of Einstein and Gauss-Bonnet gravities investigated in 
[7] and [13], respectively, we consider the case $p=n-3$. First, we consider the sevendimensional solutions of third order Lovelock gravity. In this case

$$
\begin{aligned}
& \lim _{r \rightarrow 0} f(r, v)=2 \eta_{0}, \\
& \lim _{r \rightarrow 0} \dot{f}=-\frac{4}{3}\left(\frac{3 M_{0} v_{0}}{\alpha_{3}}\right)^{1 / 3},
\end{aligned}
$$

and therefore the radial null geodesic equation near $r=v=0$ can be written as

$$
\frac{d^{2} r}{d \lambda^{2}}+\frac{2}{3 \eta_{0}^{2}}\left(\frac{3 M_{0} v_{0}}{\alpha_{3}}\right)^{1 / 3}\left(\frac{d r}{d \lambda}\right)^{2} \simeq 0
$$

with the solution

$$
r \simeq \frac{3 \eta_{0}^{2}}{2}\left(\frac{\alpha_{3}}{3 M_{0} v_{0}}\right)^{1 / 3} \ln (\lambda+1) .
$$

Using Eqs. (19), (21), (22) and (23), one finds that the limit of $\lambda \Phi$ is positive while the limit of $\lambda^{2} \Phi$ is zero as $\lambda$ goes to zero, and therefore only LFC is satisfied along a radial null geodesic. That is, along the radial null geodesics the strong curvature condition is not satisfied. Thus, the singularity of seven-dimensional third order Lovelock gravity is weaker than that of Vaidya-type solutions of Einstein gravity [7], while it has the strength as in the case of Gauss-Bonnet gravity [13].

Second, we consider $n \geq 8$ for which the limit of $f(r, v)$ is:

$$
\lim _{r \rightarrow 0} f(r, v)=1,
$$

Using Eq. (11), one obtains $v \simeq 2 r$ for small $r$ and therefore the limit of $\dot{f}$ becomes

$$
\lim _{r \rightarrow 0} \dot{f}=-\left(\frac{2^{n} M_{0} r}{9 \alpha_{3}}\right)^{1 / 3}=0 .
$$

In this case, one can show that the limits of both $\lambda \Phi$ and $\lambda^{2} \Phi$ are zero as $\lambda \rightarrow 0$. In other words, neither the SCC nor the LFC is satisfied, and therefore the third order Lovelock term weakens the strength of the singularity.

\section{B. $p=n-4$ case:}

Again, it is easy to show that along the radial null geodesics only the LFC is satisfied for a seven-dimensional solution. For $n \geq 8$, the radial null geodesic equation near $r=v=0$ can be written as

$$
\frac{d^{2} r}{d \lambda^{2}}+\frac{2 p}{3}\left(\frac{3 M_{0}}{\alpha_{3}}\right)^{1 / 3}\left(\frac{d r}{d \lambda}\right)^{2} \simeq 0
$$


with the solution

$$
r \simeq \frac{3}{2 p}\left(\frac{\alpha_{3}}{3 M_{0}}\right)^{1 / 3} \ln (\lambda+1) .
$$

Using Eqs. (19), (24), (25) and (26), one finds that the limit of $\lambda \Phi$ is positive while the limit of $\lambda^{2} \Phi$ is zero as $\lambda$ goes to zero, and therefore only the LFC is satisfied along a radial null geodesic.

\section{CLOSING REMARKS}

Considering the spherically symmetric gravitational collapse of a null dust fluid in $n \geq 7$ dimensions, we have obtained an exact solution in third order Lovelock gravity with four fundamental constants which is asymptotically $(\mathrm{A}) \mathrm{dS}$ or flat depending on the choice of the cosmological constant. This solution reduces to the Vaidya-type solution of Gauss-Bonnet gravity [13] as the third order Lovelock term is turned off, and reduces to the $n$-dimensional Vaidya-type solution of Einstein gravity for $\alpha_{1}=\alpha_{2}=0$ [7]. We applied the solution to the situation in which a null dust fluid radially injects into an initially flat region with the rate $M(v)=M_{0} v^{p}$, and investigated the effects of the third order Lovelock term on the final fate of the gravitational collapse. We found that, as in the case of Gauss-Bonnet gravity, a naked singularity is inevitably formed. In the general relativistic case, a naked singularity will form only when $M_{0}$ takes a sufficiently small value, and therefore turning on the Lovelock terms worsens the situation from the viewpoint of CCH. Furthermore, as in the case of GaussBonnet gravity, the third order Lovelock term changes the nature of the singularity and the whole picture of gravitational collapse drastically. The picture of the gravitational collapse for $n=7$ is quite different from that for $n \geq 8$, as well as the general relativistic case for $n \geq 4$ and the Gauss-Bonnet case for $n \geq 6$, a massless ingoing null naked singularity is formed. On the other hand, for the special case $n=7$, as in the case of five-dimensional Gauss-Bonnet gravity, a massive timelike naked singularity is formed. As can be seen from the result of Ref. [14] and this paper, the nature of the naked singularity is quite different for $n=2 i_{\max }+1$ and $n>2 i_{\max }+1$, where $i_{\max }+1$ is the largest order of Lovelock term. Thus, we conjecture that the naked singularity is massive in $n=2 i_{\max }+1$, while it is massless in higher dimensions.

Although naked singularities are inevitably formed in third order Lovelock gravity, the strength of the singularity is different in the cases of Einstein, Gauss-Bonnet, and third order 
Lovelock gravities. In seven dimensions, the LFC is satisfied for the solutions of GaussBonnet and third order Lovelock gravities, while the SCC is satisfied in Einstein gravity. Thus, the strength of singularities of seven-dimensional solutions of Gauss-Bonnet and third order Lovelock gravities is weaker than that of Einstein gravity. In $n \geq 8$ dimensions for $p=n-3$, the solution satisfies neither the LFC nor the SCC in third order Lovelock gravity, while the LFC is satisfied in Gauss-Bonnet gravity for the $p=n-3$. Thus, in $n \geq 8$ dimensions, turning on the third order Lovelock gravity weakens the strength of the naked singularity for the $p=n-3$ case. We also considered the strength of singularity in third order Lovelock gravity for $p=n-4$, and found that the LFC is satisfied along a radial null geodesic in $n \geq 8$ dimensions. Thus, we conjecture that the higher-order Lovelock terms weaken the strength of the singularity for the mass function $M=M_{0} v^{n-3}$. These facts show that the third order Lovelock term invites some new features of the gravitational collapse of a null dust fluid into the game. Thus, it is worth investigating the effects of higher curvature terms on the final fate of gravitational collapse.

\section{Acknowledgments}

This work has been supported by Research Institute for Astrophysics and Astronomy of Maragha. We are very grateful to anonymous referee for useful comments.

\section{APPENDIX A}

In this appendix, we calculate the quasilocal mass in third order Lovelock gravity. The Misner-Sharp mass which can be identified as the quasilocal mass has been introduced in [21] for Einstein gravity and in [14] for Gauss-Bonnet gravity. The metric of an $n$ dimensional spacetime $\left(M^{n}, g_{\mu \nu}\right)$ which is a warped product of an $(n-2)$-sphere $S^{n-2}$ and a two-dimensional orbit spacetime $\left(M^{2}, g_{a b}\right)$ under the isometries of $S^{n-2}$ may be written as

$$
g_{\mu \nu} d x^{\mu} d x^{\nu}=g_{a b}(y) d y^{b} d y^{b}+r^{2}(y) d \Omega^{2},
$$

where $a, b=0,1 ; i, j=2 \ldots(n-1)$, and $r$ is a scalar on $\left(M^{2}, g_{a b}\right)$ with $r=0$ defining its boundary. Using the method of Ref. [22] for the calculation of the quasilocal mass in 
Lovelock gravity one obtains:

$$
\begin{aligned}
\mathcal{M} & =\frac{(n-2) V_{n-2} r^{n-7}}{2 \kappa_{n}^{2}}\left\{-\Lambda r^{6}+r^{4}\left[1-(D r)^{2}\right]+\alpha_{2} r^{2}\left[1-(D r)^{2}\right]^{2}+\frac{\alpha_{3}}{3}\left[1-(D r)^{2}\right]^{3}\right\} \\
& \equiv \frac{(n-2) V_{n-2}}{2 \kappa_{n}^{2}} M
\end{aligned}
$$

where $\alpha_{i}$ 's are defined in terms of the Lovelock coefficients $\alpha_{i}^{\prime}$ in Sec. (II), $D_{a}$ is a metric compatible linear connection on $\left(M^{2}, g_{a b}\right),(D r)^{2}=g^{a b}\left(D_{a} r\right)\left(D_{b} r\right)$, and $V_{n-2}$ is the area of the $(n-2)$-sphere. It is worth mentioning that the mass (27) is consistent with the quasilocal mass proposed in Ref. [22]. Also, it is a matter of straightforward calculations to show that Conjecture 2 of Ref. [22] is true for third order Lovelock gravity. The mass given by Eq. (27) for the metric (5) reduces to

$$
M=r^{n-7}\left\{-\Lambda r^{6}+r^{4}[1-f(v, r)]+\alpha_{2} r^{2}[1-f(v, r)]^{2}+\frac{\alpha_{3}}{3}[1-f(v, r)]^{3}\right\} .
$$

Solving for $f(v, r)$, one obtains the metric function given in Eq. (9) which shows that the mass of Eq. (9) is equal to the quasilocal mass given by Eq. (27).

\section{APPENDIX B}

In this appendix, we review the definitions of different types of trapping horizons, and then consider the problem in third order Lovelock gravity. To do this, it suitable to write the line element in the double-null coordinate as

$$
d s^{2}=-2 e^{-f} d \zeta^{+} d \zeta^{-}+r^{2} d \Omega^{2}
$$

where $f$ and $r$ are functions of the null coordinates $\left(\zeta^{+}, \zeta^{-}\right)$. The null vectors $\partial / \partial \zeta^{ \pm}$will be assumed future-pointing. The expansions may be defined by

$$
\theta_{ \pm}=(n-2) r^{-1} \partial_{ \pm} r
$$

where $\partial_{ \pm}$denotes the coordinate derivative along $\zeta^{ \pm}$. The expansions measure whether the light rays normal to a sphere are diverging $(\theta>0)$ or converging $(\theta<0)$, or equivalently, whether the area of the spheres is increasing or decreasing in the null directions [16]. Note that the signs of $\theta_{ \pm}$are geometrical invariants, but their actual values are not. A compact spatial $(n-2)$-surface is said to be (i) trapped if $\theta_{+} \theta_{-}>0$, (ii) untrapped if $\theta_{+} \theta_{-}<0$, and (iii) marginal if $\theta_{+} \theta_{-}=0$. Without loss of generality, one can set $\theta_{+}=0$ at a marginal 
surface, then it is future if $\theta_{-}<0$, past if $\theta_{-}>0$, bifurcating if $\theta_{-}=0$, outer if $\partial_{-} \theta_{+}<0$, inner if $\partial_{-} \theta_{+}>0$, and degenerate if $\partial_{-} \theta_{+}=0$. The closure of a hypersurface foliated by future or past, outer or inner marginal surfaces is called a (nondegenerate) trapping horizon [16, 23].

The $\zeta \zeta$-component of the field equation is

$$
\left(\partial_{ \pm}^{2} r+\partial_{ \pm} f \partial_{ \pm} r\right)\left[1+\frac{2 \alpha_{2}}{r^{2}}\left(1+2 e^{f} \partial_{+} r \partial_{-} r\right)+\frac{\alpha_{3}}{r^{4}}\left(1+2 e^{f} \partial_{+} r \partial_{-} r\right)^{2}\right]=-\frac{\kappa_{n}^{2}}{n-2} T_{ \pm \pm}
$$

which reduces to

$$
2 \partial_{ \pm} \theta_{ \pm}\left[1+\frac{2 \alpha_{2}}{r_{h}^{2}}+\frac{\alpha_{3}}{r_{h}^{4}}\right]=-\frac{\kappa_{n}^{2}}{n-2} T_{ \pm \pm}
$$

on the trapping horizon. The above discussion shows that, if the null energy condition holds, the trapping horizon is a future outer trapping horizon.

Finally, we consider the relation between the null energy condition and the null convergence condition along the radial null vectors. It is a matter of calculation to show that for a radial null vector $k^{\mu} \partial / \partial x^{\mu}=k^{+} \partial / \partial \zeta^{+}+k^{-} \partial / \partial \zeta^{-}$, one has

$$
\kappa_{n}^{2} T_{\mu \nu} k^{\mu} k^{\nu}=R_{\mu \nu} k^{\mu} k^{\nu}\left\{1+\frac{\alpha_{2}^{2}}{\alpha_{3}}\left[C^{1 / 3}(r, v)-\gamma^{1 / 3} C(r, v)^{-1 / 3}\right]^{2}\right\}
$$

for our solution (9). This equation shows that, as in the case of Gauss-Bonnet gravity for solutions with a general relativity limit [23], the null convergence condition $R_{\mu \nu} k^{\mu} k^{\nu} \geq 0$ is satisfied provided the null energy condition $T_{\mu \nu} k^{\mu} k^{\nu}>0$ holds.

[1] J. R. Oppenheimer and H. Snyder, Phys. Rev. 56, 455 (1939).

[2] S. W. Hawking and G. F. R. Ellis, The Large Scale Structure of Spacetime (Cambridge University Press, Cambridge, England, 1973).

[3] R. Penrose, Riv. del Nuovo Cim. 1, 252 (1969); in General Relativity, an Einstein Centenary Volume, edited by S. W. Hawking and W. Israel (Cambridge University Press, England, 1979).

[4] P. C. Vaidya, Proc. Indian Acad. Sci. A33, 264 (1951); Reprinted, Gen. Rel. Grav. 31, 119 (1999).

[5] Y. Kuroda, Prog. Theor. Phys. 72, 63 (1984); I. H. Dwivedi and P. S. Joshi, Class. Quantum Grav. 6, 1599 (1989); P. S. Joshi and I. H. Dwivedi, J. Math. Phys. 32, 2167 (1991); Gen. 
Rel. Grav. 24, 129 (1992); J. P. S. Lemos, Phys. Rev. Lett. 68, 1447 (1992); Phys. Rev. D 59, 044020 (1999).

[6] M. D. Roberts, Gen. Rel. Grav. 21, 907 (1989); S. M. Wagh and S. D. Maharaj, ibid. 31, 975 (1999); A. K. Dawood and S. G. Ghosh, Phys. Rev. D 70, 104010 (2004).

[7] N. Dadhich and S. G. Ghosh, Phys. Lett. B518, 1 (2001); S. G. Ghosh and N. Dadhich, Phys. Rev. D 64, 047501 (2001).

[8] D. G. Boulware and S. Deser, Phys. Rev. Lett. 55, 2656 (1985); J. T. Wheeler, Nucl. Phys. B268, 737 (1986).

[9] Y. M. Cho and I. P. Neupane, Phys. Rev. D 66, 024044 (2002); I. P. Neupane, ibid. 67, 061501(R) (2003); ibid.69, 084011 (2004); R. G. Cai, ibid. 65084014 (2002); R. G. Cai and Q. Guo, ibid. 69, 104025 (2004); T. Torii and H. Maeda, ibid. 71, 124002 (2005); M. H. Dehghani, ibid. 69, 064024 (2004); M. H. Dehghani and R. B. Mann, ibid. 72, 124006 (2005); M. H. Dehghani and S. H. Hendi, ibid. 73, 084021 (2006); M. H. Dehghani, G. H. Bordbar and M. Shamirzaie, ibid. 74, 064023 (2006); M. H. Dehghani and S. H. Hendi, Int. J. Mod. Phys. D 16, 1829 (2007); A. Padilla, Class. Quantum Grav. 20, 3129 (2003); N. Deruelle, J. Katz, and S. Ogushi, ibid. 21, 1971 (2004); M. Cvetic, S. Nojiri, and S. D. Odintsov, Nucl. Phys. B628, 295 (2002).

[10] M. H. Dehghani, M. Shamirzaie, Phy. Rev. D 72, 124015 (2005).

[11] M. H. Dehghani, R. B. Mann, Phy. Rev. D 72, 124006 (2005); M. H. Dehghani, N. Alinejadi and S. H. Hendi, ibid. 77, 104025 (2008); S. H. Hendi and M. H. Dehghani, Phys. Lett. B666, $116(2008)$.

[12] S. Nojiri, S. D. Odintsov and M. Sami, Phys. Rev. D 74, 046004 (2006); E. Elizalde et. al., Eur. Phys. J. C53, 447 (2008).

[13] T. Kobayashi, Gen. Rel. Grav. 37, 1869 (2005).

[14] H. Maeda, Phys. Rev. D 73, 104004 (2006).

[15] M. Nozawa and H. Maeda, Class. Quantum Grav. 23, 1779 (2006).

[16] S. A. Hayward, Phys. Rev. D 49, 6467 (1994).

[17] D. Christodoulou, Commun. Math. Phys. 93171 (1984).

[18] C. J. S. Clarke and K. Krolak, J. Geom. Phys. 2, 127 (1985).

[19] F. J. Tipler, Phys. Lett. A64, 8 (1977); F. J. Tipler, C. J. S. Clarke, and G. F. R. Ellis, in General Relativity and Gravitation, edited by A. Held (Plenum, New York, 1980). 
[20] A. Krolak, J. Math. Phys. 28, 138 (1987).

[21] C. W. Misner and D. H. Sharp, Phys. Rev. 136, B571 (1964); S. A. Hayward, Phys. Rev. D 53, 1938 (1996).

[22] H. Maeda and M. Nozawa, Phys. Rev. D 77, 064031 (2008); H. Maeda, Phys. Rev. D 78, 041503(R) (2008).

[23] M. Nozawa and H. Maeda, Class. Quantum Grav. 25, 055009 (2008). 